\title{
Partial Clustering in Binary Two-Dimensional Colloidal Suspensions
}

\author{
Norman Hoffmann, ${ }^{1}$ Florian Ebert, ${ }^{2}$ Christos N. Likos, ${ }^{1}$ Hartmut Löwen, ${ }^{1}$ and Georg Maret ${ }^{2}$ \\ ${ }^{1}$ Institut für Theoretische Physik II, Heinrich-Heine-Universität Düsseldorf, Universitätsstraße 1, D-40225 Düsseldorf, Germany \\ ${ }^{2}$ Fachbereich für Physik, Universität Konstanz, D-78457 Konstanz, Germany
}

(Received 28 March 2006; published 15 August 2006)

\begin{abstract}
Strongly interacting binary mixtures of superparamagnetic colloidal particles confined to a twodimensional water-air interface are examined by theory, computer simulation, and experiment. The mixture exhibits a partial clustering in equilibrium: in the voids of the matrix of unclustered big particles, the small particles form subclusters with a spongelike topology which is accompanied by a characteristic small-wave vector peak in the small-small structure factor. This partial clustering is a general phenomenon occurring for strongly coupled negatively nonadditive mixtures.
\end{abstract}

DOI: 10.1103/PhysRevLett.97.078301

Self-organization processes in suspensions of colloidal particles are of key relevance for the construction of nanodevices with desired mechanical, rheological, and optical properties. In particular, colloidal aggregates or clusters can be used as supramolecular building blocks, motivated by the quest for photonic band-gap materials [1], molecular sieves [2,3], and microfilters with desired porosity [4]. Colloidal cluster formation is mainly controlled by the effective interparticle forces [5]. Recently, it was predicted that a combination of short-ranged attractive and longranged repulsive forces [6,7] or ramplike repulsions [8] can lead to equilibrium clusters for the case of hard colloids, whereas particular classes of ultrasoft colloids can show clustering even in the complete absence of attraction [9]. The cluster morphology critically depends on the details of the interaction, being, e.g., spherical or wormlike with an internal spiral structure [10,11]. The equilibrium clustering has been confirmed in recent experiments [1214] of one-component weakly charged suspensions with polymer additives.

In this Letter, we address equilibrium clustering in twocomponent suspensions of big and small colloidal particles. Our motivation is twofold: first, any realistic sample consists of several components and hence considering a binary mixture is an important step towards a control of novel composite materials. Second, mixtures exhibit much richer behavior than their one-component counterparts. Specifically, we consider here a binary system of superparamagnetic colloidal particles that are confined to a planar water-air interface and exposed to an external magnetic field perpendicular to the interface. The magnetic field induces a magnetic dipole moment on the particles, resulting in an effective repulsion between all parallel oriented dipole moments, which scales with the inverse cube of the particle distance. This enables a direct comparison between theories and computer simulations based on a pairwise dipole-dipole interaction potential and renders these suspensions into an ideal model system [15], allowing thereby to additionally confront the theoretical findings with direct experimental data. The advantage of
PACS numbers: 82.70.Dd, 61.20.-p, 61.20.Ja, 64.75.+g

the availability of experimental systems has been exploited to study two-dimensional melting in the one-component system [16,17] and for the study of the dynamics [18] and the glass transition [19] of the binary system. A quite similar system of electrical dipoles for binary colloid monolayers has been studied recently be means of molecular dynamics simulations [20] but attention there was focused exclusively on the formation of regular lattices for rational values of the composition. Yet, cluster formation for this system has not been studied to date. The system at hand presents a host of novel features: it is a two-dimensional system, it has clearly defined interactions between all its constituent particles, which take a simple form and are steered by a single and readily tunable external field; it is experimentally realizable and allows direct comparison with theory; and, finally, it is apt to direct visual observation.

We find that colloidal clustering is specific to the components in the repulsive mixture: subclustering is possible where only the small particles form clusters but the big ones are unclustered. We trace the origins of the phenomenon to the negative nonadditivity [21] of the mixture, $\Delta<0$, defined as follows. Let $v_{i j}(r), i, j=1,2$ be the three interaction potentials between all species and introduce the corresponding Barker-Henderson effective hard core diameters $\sigma_{i j}=\int_{0}^{\infty} d r\left\{1-\exp \left[-v_{i j}(r) / k_{B} T\right]\right\}$, with Boltzmann's constant $k_{B}$ and the absolute temperature $T$. Then, $\Delta=2 \sigma_{12}-\left(\sigma_{11}+\sigma_{22}\right)$. The partial clustering effect should therefore be more generic to negatively nonadditive binary mixtures of, e.g., charged colloids [22] or star polymers and linear chains [23], where, in addition, the relationship $v_{22}(r)<v_{12}(r)<v_{11}(r)$ is fulfilled, as in the case at hand. However, partial clustering is absent in strictly additive $(\Delta=0)$ hard sphere mixtures and in colloid-polymer mixtures, which are positively nonadditive $(\Delta>0)$. The structural signature of this phenomenon is the appearance of an additional small-wave-vector prepeak in the small-small structure factor correlations, which does not show up in the big-big structure factor. Based on a topological analysis using the Euler characteristic, we 
show that the small particles have a nontrivial, spongelike structure. We base our findings on liquid-integral equation theory, computer simulations, image analysis, and video microscopy data of real samples, finding very good agreement between all.

In our theoretical model, we consider two different species of colloidal particles moving freely in a twodimensional plane, which corresponds to the experiment (see Fig. 1). Each component is characterized by its partial density $\rho_{i}$ and its susceptibility $\chi_{i}, i=1,2$. The external magnetic field $\mathbf{B}$ standing perpendicular to the plane, induces in each particle a magnetic moment $m_{i}=\chi_{i} B, i=$ 1,2 . The particles are superparamagnetic, i.e., the magnetic dipole of each species perfectly aligns with the external field. We model all particles as pointlike but refer thereafter to the particles having the larger susceptibility as the "big" (species 1) and those with smaller susceptibility as "small" (species 2). The particles interact via purely repulsive dipole-dipole pair potentials $\beta v_{i j}(x)=\Gamma_{i j} / x^{3}, i$, $j=1,2$, where $\beta=\left(k_{B} T\right)^{-1}$ and $x$ denotes the distance between any two particles scaled over the average interparticle separation between big particles, $x \equiv r \sqrt{\rho_{1}}$. The interaction strengths $\Gamma_{i j}$ are given as $\Gamma_{i j}=\beta \chi_{i} \chi_{j} B^{2} / a_{11}^{3}$, $\alpha_{11}=1 / \sqrt{\rho_{1}}$. The system is fully characterized by three quantities: the density ratio $\rho_{2} / \rho_{1}$, the susceptibility ratio $\chi_{2} / \chi_{1}<1$, and one of the three interaction strengths, which we pick to be $\Gamma_{11}$.

We have performed Monte Carlo computer simulations [24] in a square box with periodic boundary conditions. Finite-size effects were carefully checked for by varying the box size from 500 to 2000 particles. Additionally, we solved the binary Ornstein-Zernike relation [25] in two spatial dimensions, using the Rogers-Young (RY) closure [26]. The RY closure gives reliable results in comparison to the simulation for all parameter combinations investigated, see representative results in Fig. 2. Thus, we rely on this closure to calculate the structure of the fluid. In Fig. 2, the clustering of the small particles is manifest by an additional small-wave-vector peak in the structure factor $S_{22}(k)$ whereas $S_{11}(k)$ is devoid of any such signature. Hence, the formation of small-particles subclusters in absence of big

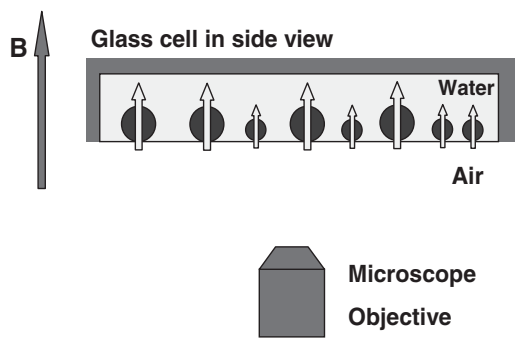

FIG. 1. Schematic view of the setup: binary mixture of superparamagnetic colloidal particles at an air-water interface in an external magnetic field $\mathbf{B}$ perpendicular to the plane. particle clusters is witnessed by structural information in reciprocal space.

The experimental system is fully described by the theoretical model introduced above and is explained in detail elsewhere [15,16]. We use superparamagnetic particles [27] with susceptibilities $\chi_{1}=6.2 \times 10^{-11} \mathrm{Am}^{2} / \mathrm{T}$ and $\chi_{2}=6.6 \times 10^{-12} \mathrm{Am}^{2} / \mathrm{T}$, which are suspended in a free-hanging, flat water droplet attached to a top-sealed glass ring (diameter $=8 \mathrm{~mm}$, see Fig. 1). The particles are made from porous polystyrene spheres that are doped with small $\mathrm{Fe}_{2} \mathrm{O}_{3}$ clusters to make them superparamagnetic and are further sealed with an epoxy layer. Stabilization with sodium dodecyl sulfate prevents the particles from aggregation. Because of high mass density $\left(d_{1}=1.3 \mathrm{~kg} / \mathrm{dm}^{3}\right.$, $d_{2}=1.5 \mathrm{~kg} / \mathrm{dm}^{3}$ ), both types of particles are pinned down to the water-air interface by gravity and form an ideal, twodimensional monolayer of binary dipoles. The relatively small gravitational lengths of $l_{1}=8 \mathrm{~nm}$ and $l_{2}=62 \mathrm{~nm}$ for big and small particles, respectively, compared to the particle diameters of $4.7 \mu \mathrm{m}$ and $2.8 \mu \mathrm{m}$ ensure an almost perfect realization of a 2D system. The flatness of the interface can be controlled in the range of less than $1 \mu \mathrm{m}$. Inclination control of the whole setup guarantees a nearly horizontal alignment of the flat surface, ruling out the occurrence of any density gradients in the sample. We control the interaction strengths $\Gamma_{i j}$ between the dipoles by applying an external magnetic field up to $4 \mathrm{mT}$, perpendicular to the interface. The dipole interaction dominates all other interactions in this colloidal system [15]. The sample was conserved for months and measurements were taken for a duration of up to 24 hours. All necessary data were recorded by video microscopy. Typically, about 1300 particles were observed in a box of $689 \mu \mathrm{m} \times$ $505 \mu \mathrm{m}$, with a total amount of $10^{6}$ particles in the whole sample. The density ratio $\rho_{2} / \rho_{1}$ was varied between 0.67 and 1. Local and statistical properties of the sample are gathered at a rate of about 1 frame/second on all relevant time and length scales.

A comparison between the theoretical and experimental structure factors is shown in Fig. 3, pertaining to susceptibility ratio $\chi_{2} / \chi_{1}=0.1$ and corresponding to the real experimental situation. The representative results are
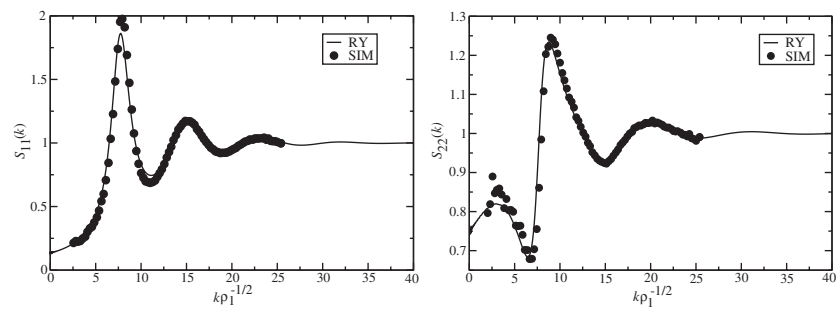

FIG. 2. Partial structure factors $S_{11}(k)$ for the big particles and $S_{22}(k)$ for the small particles. Computer simulation results are compared to the RY closure for the following parameter combination: $\Gamma_{11}=4.0, \rho_{2} / \rho_{1}=0.5, \chi_{2} / \chi_{1}=0.5$. 

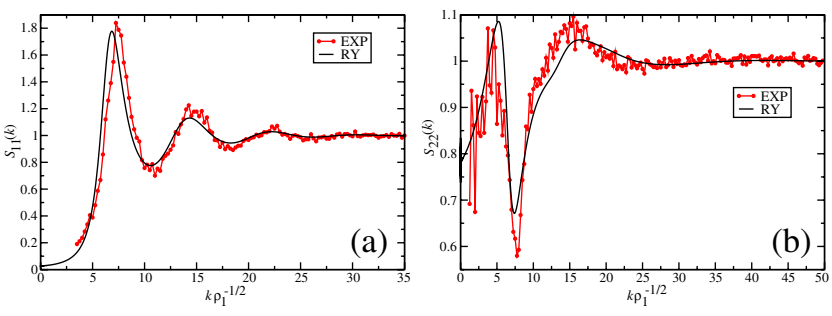

FIG. 3 (color online). Partial structure factors $S_{11}(k)$ for the big particles and $S_{22}(k)$ for the small particles. Experimental results (EXP) are compared to theoretical ones (RY) for the parameters: $\Gamma_{11}=4.05, \rho_{2} / \rho_{1}=0.89, \chi_{2} / \chi_{1}=0.1$.

shown here for density ratio $\rho_{2} / \rho_{1}=0.89$ and coupling constant $\Gamma_{11}=4.05$. Very good agreement between theory and experiment is achieved, supporting the modeling of the system by means of dipolar interactions exclusively; evidently, all other residual forces in the colloidal suspension are much weaker and can be neglected. A prepeak is seen in the small-particles structure factor $S_{22}(k)$, whose height is comparable with that of the second peak due to the small value of $\chi_{2}$. Indeed, as the second peak arises from pure small-small interactions, its height is suppressed since the latter scale as $\chi_{2}^{2}$. The prepeak trivially disappears in both limits $\chi_{2} / \chi_{1} \rightarrow 0$ and $\chi_{2} / \chi_{1} \rightarrow 1$.

Further evidence of the subclustering phenomenon is provided by real-space data. In Fig. 4 we show experimental and simulation snapshots of the system under consideration. We observe a cluster structure of the smaller particles that perfectly fits in the voids shaped by the big particles. To better quantify the morphology of the spatial patterns, we employ tools from integral geometry, and, in particular, the Euler characteristic $E$, which describes the topology of the pattern [28]. We have discretized the snapshot by superimposing a grid on the picture, surround each
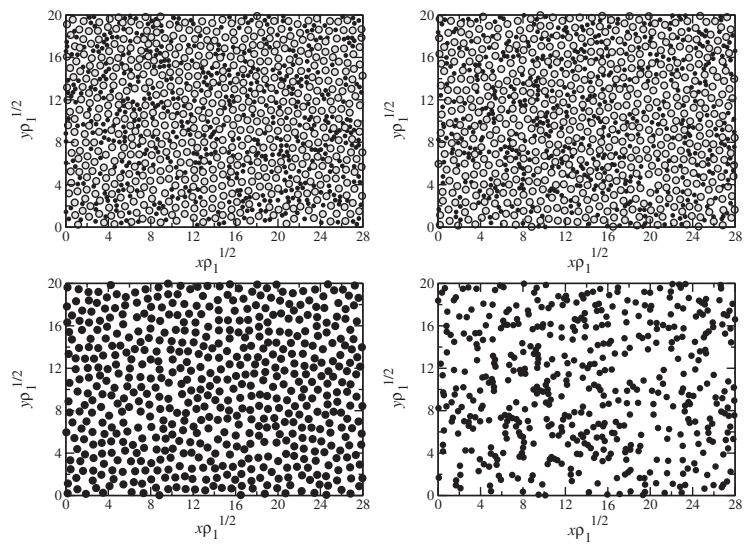

FIG. 4. Snapshots of the binary magnetic mixture from experiment and simulation for the parameter combination $\Gamma_{11}=4.05$, $\rho_{2} / \rho_{1}=0.89, \chi_{2} / \chi_{1}=0.1$. The big particles are denoted gray and the small ones black. Clockwise from the upper left panel: experiment, simulation (both species), simulation (small particles only), and simulation (big particles only). particle by circle, and gradually increase the (cover) radius $R_{0}$ of the latter. The Euler characteristic was then calculated on the discrete lattice [28]. The resulting spatial pattern consists then of a number $C$ of connected components and a number $H$ of holes and $E=C-H$ [28].

In Fig. 5(a), we show the $E$ curves of the two components of a binary mixture, compared to a one-component and an ideal gas system. The underlying snapshots are taken from simulation data. The Euler characteristic of the small component tends to zero much more slowly than the ideal gas curve. For the ideal gas, the particles are more or less evenly spread over the area, so the vacant space between the particles can be filled much faster than in the two-component case where the interaggregate space between the small particles is larger. A comparison between the big component and the one-component system reveals that the Euler characteristic of the former becomes less negative and tends to zero much more slowly due to the larger voids caused by the aggregation of the small component. Figure 5(b) refers to the parameter combination of the experimental sample. There, the $E$ versus $R_{0}$ curves from simulation and experiment are compared, showing very good agreement. The prepeak position in Fig. 3(b) points to a typical cluster size $L_{\mathrm{cl}} \sqrt{\rho_{1}} \cong 1.26$ which agrees well with the position of the most negative value of $E_{2}$ from Fig. 5(b) and is consistent with the large holes left there when the covering circle radius $R_{0} \cong$ $L_{\mathrm{cl}} / 2$. The partial clustering is caused by the interplay between the successively stronger repulsive interactions $v_{22}(r)<v_{12}(r)<v_{11}(r)$, which preclude macrophase separation and favor the nesting of small particles in the voids of the big ones.

We have studied equilibrium cluster formation in a twocomponent colloidal model system. We find partial clustering: only the small particles show a clustering, as witnessed by a first peak in their structure factor while the big particles do not exhibit any peak on this scale. The cluster structure does not consist of well-separated isolated clusters with a well-defined geometrical shape, such as circular or lamellar, which form for interactions with a short-range
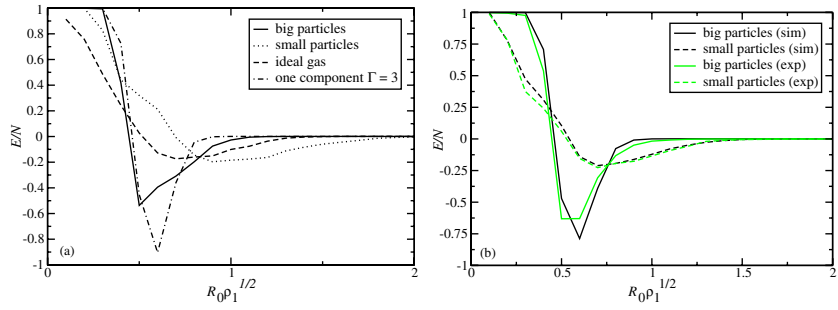

FIG. 5 (color online). (a) Euler characteristic per particle, $E / N$, from simulation for a binary magnetic mixture and $\Gamma_{11}=$ 3.0, $\rho_{2} / \rho_{1}=0.5, \chi_{2} / \chi_{1}=0.5$. (b) Comparison of the theoretical (black lines) and experimental (gray lines) of the same quantity for the parameters $\Gamma_{11}=4.05, \rho_{2} / \rho_{1}=0.89, \chi_{2} / \chi_{1}=$ 0.1 . Solid lines: big particles; dashed lines: small particles. 
attraction and a long-range repulsion [7]. Rather, it possesses a percolating spongelike topology, as signaled by a considerably negative $E$ value for large covering circles. We presented results for $\chi_{2} / \chi_{1}=0.5$ and 0.1 but the subclustering phenomenon holds for a broad range of parameter combinations and, in particular, up to $\chi_{2} / \chi_{1}=$ 0.7 and for a density range at least as broad as $0.1 \leq$ $\rho_{2} / \rho_{1} \leq 2.5$, in which we performed additional calculations.

The subclustering behavior is more general and not just limited to paramagnetic colloids. Similar effects are encountered in binary star polymer mixtures [29]. Our experimentally realizable system offers a clean and reproducible example of this phenomenon which has not received due attention in two spatial dimensions up to now. The common feature of systems exhibiting partial clustering is that they possess a negative nonadditivity, i.e., if their cross interaction is less repulsive than the sum of the two direct interactions. Partial clustering, however, is absent in additive hard sphere mixtures [30]. In systems with positive nonadditivity like the Asakura-Oosawa-Vrij model [31], partial clustering was never observed, due to the attractions induced by the polymers, which drive macrophase separation. It would be interesting to study the viscosity and the propagation of light in partial clustered structures in order to extract their specific material properties. It is further tempting to shock freeze the clustered structure and use it as a bicontinuous device of controlled, random nanoporosity.

We thank D. Levesque for helpful discussions, C. Mayer for providing unpublished data, and R. Hund for providing raw data of the experiments. This work has been supported by the DFG within the SFB-TR6, Project Sections C2 and C3.

[1] V. N. Manoharan et al., Science 301, 483 (2003).

[2] J. Kecht et al., Langmuir 20, 5271 (2004).

[3] C. Lutz et al., Phys. Rev. Lett. 93, 026001 (2004).
[4] F. Yan and W. A. Goedel, Chem. Mater. 16, 1622 (2004); Nano Lett. 4, 1193 (2004).

[5] P.N. Pusey, in Liquids, Freezing and Glass Transition, edited by J.-P. Hansen, D. Levesque, and J. Zinn-Justin, Proceedings of the Les Houches Summer School, Session LI (North-Holland, Amsterdam, 1991).

[6] R. P. Sear and W. M. Gelbart, J. Chem. Phys. 110, 4582 (1999).

[7] A. Imperio and L. Reatto, J. Phys. Condens. Matter 16, S3769 (2004).

[8] G. Malescio and G. Pellicane, Nat. Mater. 2, 97 (2003).

[9] B. M. Mladek et al., Phys. Rev. Lett. 96, 045701 (2006).

[10] F. Sciortino et al., Phys. Rev. Lett. 93, 055701 (2004).

[11] S. Mossa et al., Langmuir 20, 10756 (2004).

[12] A. Stradner et al., Nature (London) 432, 492 (2004).

[13] A. I. Campbell et al., Phys. Rev. Lett. 94, 208301 (2005).

[14] P. J. Lu et al., Phys. Rev. Lett. 96, 028306 (2006).

[15] K. Zahn et al., Phys. Rev. Lett. 79, 175 (1997).

[16] K. Zahn et al., Phys. Rev. Lett. 82, 2721 (1999); 85, 3656 (2000).

[17] C. Eisenmann et al., Phys. Rev. Lett. 93, 105702 (2004).

[18] M. Kollmann et al., Europhys. Lett. 58, 919 (2002).

[19] H. König et al., Eur. Phys. J. E 18, 287 (2005).

[20] T. Stirner and J. Sun, Langmuir 21, 6636 (2005).

[21] R. Roth and R. Evans, Europhys. Lett. 53, 271 (2001).

[22] J. M. Méndez-Alcaraz et al., Physica (Amsterdam) A220, 173 (1995).

[23] E. Stiakakis et al., Europhys. Lett. 72, 664 (2005).

[24] M.P. Allen and D. J. Tildesley, Computer Simulation of Liquids (Clarendon, Oxford, 1987).

[25] J.-P. Hansen and I. R. McDonald, Theory of Simple Liquids (Academic, New York, 1986), 2nd ed.

[26] F. J. Rogers and D. A. Young, Phys. Rev. A 30, 999 (1984).

[27] DYNABEADS M-450, uncoated; Deutsche Dynal GmbH, Postfach 111965, D-20419 Hamburg, Germany.

[28] C. N. Likos et al., J. Chem. Phys. 102, 9350 (1995).

[29] C. Mayer et al. (to be published).

[30] N. W. Ashcroft and D. C. Langreth, Phys. Rev. 156, 685 (1967).

[31] H. N. W. Lekkerkerker et al., Europhys. Lett. 20, 559 (1992). 